Décadrages Décadrages

cinéma, à travers champs Cinéma, à travers champs

$6 \mid 2005$

Train et cinéma

\title{
Du pont tournant à la vue panoramique
}

Les dispositifs ferroviaires de Richard Serra et de Ken Jacobs

\section{François Bovier}

\section{OpenEdition}

Journals

Édition électronique

URL : https://journals.openedition.org/decadrages/485

DOI : $10.4000 /$ decadrages.485

ISSN : 2297-5977

Éditeur

Association Décadrages

Édition imprimée

Date de publication : 10 octobre 2005

Pagination : 36-47

ISBN : 978-2-9700582-2-9

ISSN : 2235-7823

Référence électronique

François Bovier, «Du pont tournant à la vue panoramique », Décadrages [En ligne], 6 | 2005, mis en ligne le 20 janvier 2014, consulté le 27 mars 2022. URL : http://journals.openedition.org/decadrages/ 485 ; DOI : https://doi.org/10.4000/decadrages.485 


\title{
Du pont tournant à la vue panoramique: \\ les dispositifs ferroviaires de Richard Serra
}

\author{
et de Ken Jacobs
}

par François Bovier

1 Nous pensons aux réflexions de Benjamin sur les media de reproduction et aux reprises auxquelles elles ont pu donner lieu, notamment auprès de Bürger. Voir Walter Benjamin, "L'œuvre d'art à l'époque de sa reproductibilité technique " [première version: 1935], CEuvres III, Paris, Gallimard (Folio), 2000, pp. 67-113 (traduit par Pierre Rusch) ; Peter Bürger, Theory of the Avant-Garde, Minneapolis, University of Minnesota Press, 1984 (traduit par Michael Shaw) [première édition: Theorie der AvantGarde, Suhrkamp Verlag, 1974].
A plus d'un égard, le train et le cinéma sont des appareils apparentés. En premier lieu, leur rythme est ajusté : le mouvement des bielles et le battement alternatif des roues suggèrent le défilement des photogrammes, voire le montage, comme l'expose la série d'études filmiques de Guy Sherwin, The Train Films (Grande-Bretagne, 1977-2004, environ 50', durée et formats modulables). Toutefois, la dynamique des voyages ferroviaires a pu être déclinée à travers d'autres supports d'expression, dont le paradigme littéraire demeure La prose du Transsibérien et de la petite Jehanne de France (1913) de Blaise Cendrars (qui a par ailleurs travaillé pour Gance sur La roue). En deuxième lieu, leur système optique est apparenté : les fenêtres du train cadrent un paysage qui défile, tout comme la caméra découpe une vue. Au cinéma, la relation entre ces dispositifs de cadrage et les panoramas n'a pas échappé à Max Ophuls qui met en scène leur dynamique fantasmatique d'évasion, lors d'une scène de séduction entre les protagonistes sur fond de paysages touristiques en carton pâte (Letter from an Unknown Woman, Lettre d'une inconnue, Etats-Unis, 1948). En troisième lieu, leur motricité est assimilable: le train et le cinéma sont des machines à arpenter l'espace et à renouveler notre vue. Dans Pacific 231 (France, 1949, 10'), Jean Mitry retranscrit ce dynamisme confinant à l'abstraction, en passant par l'intermédiaire de Honegger. En quatrième lieu, le train et le cinéma ont contribué à remodeler notre expérience de l'espace et du temps, provoquant une abolition relative des distances et, pour le cinéma, une fragmentation du temps. Bref, ces deux appareils, structurés selon un mode discontinu, participent à ce qui a pu être théorisé comme une expérience du choc - expérience définitoire s'il en est de la modernité et des avant-gardes $\mathbf{1}$.

Dans le champ des études cinématographiques, l'analyse se focalise surtout sur la présence du train - en tant que motif, support de vues et 
appareil épistémique - dans le cinéma des premiers temps. Pourtant, les recoupements sont patents avec le cinéma expérimental: les reprises de dispositifs ferroviaires en lien avec le cinéma des premiers temps dans les films expérimentaux et d'artistes constituent, si ce n'est un lieu commun, tout au moins une communauté de lieu. Al Razutis, qui entrecroise L'arrivée d'un train en gare de la Ciotat (Louis Lumière, France, 1895, 0' 50"), La roue (Abel Gance, France, 1924) et Spills for Thrills (production de la Warner Brothers), condense bien l'interaction qui se noue entre regard mobile, mécanicité et accidents (Lumière's Train, 1979, Canada, 9'). La rencontre entre films primitifs et expérimentaux remonte à la fin des années 1960 déjà, certains cinéastes interrogeant les modalités de constitution du sens dans les films au tournant du siècle ${ }^{2}$. Le domaine académique accuse un certain retard au regard des expérimentations des praticiens : le cinéma des premiers temps - qu'il soit ou non question de dispositifs ferroviaires - n'a fait l'objet d'études systématiques qu'à partir de 1978, si nous admettons que le colloque de Brighton fait acte de tournant dans la recherche, notamment en écartant tout préjugé téléologique. Toutefois, il faut le souligner, les intentions entre praticiens et chercheurs ou historiens divergent. Lorsque Ernie Gehr intervient sur une vue prise depuis l'avant d'un tramway au début du siècle (Eureka!, Etats-Unis, 1974, 30'), il s'agit pour lui de jouer, sans prétendre à une quelconque "objectivité scientifique», un mode de représentation primitif contre un mode de représentation institutionnel - opposition à visée axiologique qui traverse l'étude historique de Noël Burch sur la linéarisation du signifiant filmique et qui hante son essai filmique exposant différentes modalités de représentations (Correction Please, Grande-Bretagne, 1979, 52') 3. Ces pôles, que d'autres ont formalisés à travers le distinguo entre l'attraction et la narration, ne sont d'ailleurs pas fixés historiquement - ils coexistent, puis se déplacent.

Aussi naviguerons-nous entre les époques, cantonnant le cinéma des premiers temps, auquel le train semble associé, à un horizon d'attente qui parfois s'actualise - certains films expérimentaux et d'artistes reposant exhaustivement sur des sources du début du siècle, d'autres les mobilisant implicitement en tant que dispositif. Plutôt que de prétendre embrasser un champ dont il n'est pas aisé de faire le tour, nous nous concentrerons sur un motif négligé, le pont ferroviaire. Dans cette topique, nous privilégierons un film : Railroad Turnbridge (Etats-Unis, 1975-1976, 16' 30") de Richard Serra. Nous le rapporterons par la suite à deux films de Ken Jacobs, Disorient Express (Etats-Unis, 1996, 30') et The Georgetown Loop (Etats-Unis, 1996, 11'), qui constituent, selon nous, une inversion du dispositif de "surcadrage» de Serra, tout en exploitant des métrages des premiers temps.
2 Bart Testa propose une excellente synthèse de cette interaction entre cinéma expérimental et cinéma primitif (Back and Forth. Early Cinema and the Avant-Garde, Art Gallery of Ontario, Ontario, 1992).

3 Burch oppose au mode de représentation institutionnel un mode de présentation primitif avec lequel renouent les avant-gardes contemporaines (Noël Burch, La lucarne de l'infini. Naissance du langage cinématographique, Nathan (Nathan-Université), Paris, 1991 [première édition anglaise: 1990]). 


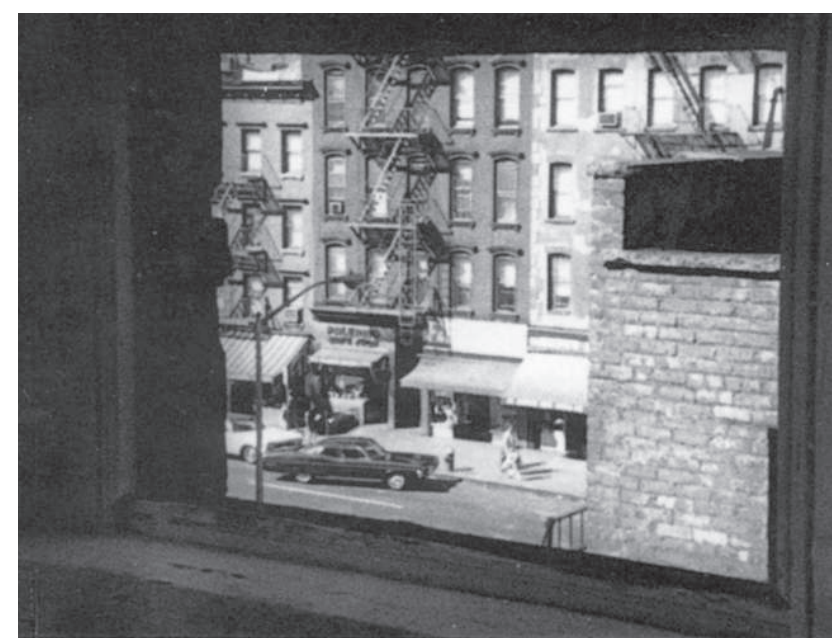

FRAME (1969)

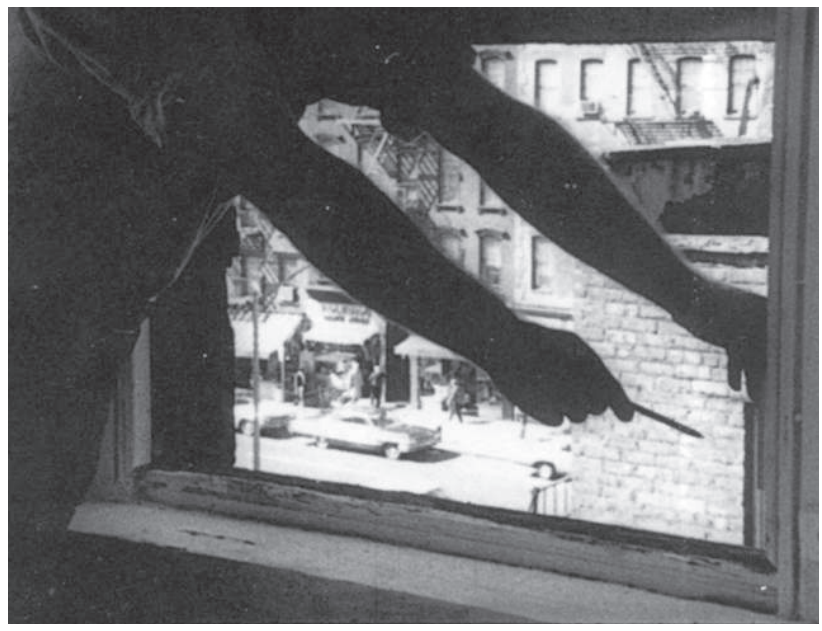

\section{Le redoublement du cadrage chez Serra}

Dans sa pratique filmique, le sculpteur post-minimaliste Serra a interrogé la question du cadrage, de la délimitation et de la mesure d'un champ de vision. Après avoir tourné des films centrés sur des gestes répétitifs, morcelant la représentation du corps (attraper au vol des morceaux de plomb qui tombent; maintenir de la main un rouleau de plomb, le bras tendu; les mains nouées, se défaire de ses liens; débarrasser de ses mains un espace encombré de copeaux d'acier), il a mis en abîme le dispositif du cadrage, avec Frame (Etats-Unis, 1969, 22') puis Railroad Turnbridge. La question du cadrage entre en résonance avec le travail sculptural de 
Serra sur le poids, la masse, la découpe et le concassage des matériaux qu'il manipule, respectant la spécificité et la dimensionnalité des sites urbains où il intervient 4 . Mais lorsqu'il interroge le support cinématographique, nous pouvons émettre la gageure qu'il se place à l'intérieur de ce champ qu'il connaît bien, assistant régulièrement aux séances de l'Anthology Film Archives dès sa fondation en 1970 et nouant des liens avec les cinéastes expérimentaux dits structurels, notamment avec Michael Snow qui, de toute évidence, influence sa pratique filmique ${ }^{5}$. Retraçons donc les modalités de mise en jeu du cadrage dans Frame et Railroad Turnbridge - en notant d'emblée que d'un film à l'autre, nous basculons de l'ordre de la démonstration théorique à la mise en acte d'un dispositif préexistant au film.

Dans Frame, un homme mesure l'espace que cadre l'objectif de la caméra, contre un mur blanc. La caméra ayant pivoté d'environ $30^{\circ}$, l'homme mesure l'espace que cadre l'objectif contre une paroi blanche, apposée à une fenêtre (le parallélépipède ainsi formé est perçu comme un rectangle bidimensionnel par le spectateur). La paroi retirée, l'homme mesure le cadre de la fenêtre, avec le même angle de $30^{\circ}$. Enfin, il mesure l'image filmée de cette fenêtre, projetée de face contre le mur. Le film met ainsi en évidence la disjonction entre ce que voit l'opérateur à travers l'objectif, la vision de l'homme qui mesure l'espace réel et le spectateur du film. Une confusion est donc entretenue entre le cadre de la caméra, la mesure d'un cadrage sur un mur et le cadre d'une fenêtre filmée sous différents angles (la fameuse ouverture sur le monde d'Alberti et de la construction en perspective, ici niée au profit tantôt d'un rectangle blanc, tantôt d'un simulacre filmique). Ce film axiomatique administre la preuve que le cinéma n'échappe pas à l'ordre de la convention et de l'illusion: s'opposant à la notion de films sculpturaux, Serra affirme la planéité de l'écran contre l'illusion du relief et de la profondeur de champ au cinéma. Comme il l'a explicitement soutenu, il n'y a «aucune possibilité de penser le cadre comme sculptural. [Celui-ci] nie la possibilité de faire l'expérience de l'espace physique réel qui, lui, est sculptural» $\boldsymbol{6}$. Nous le verrons, la performance de Ken Jacobs, From Muybridge to Brooklyn Bridge (1996), retourne littéralement cette proposition.

Dans Railroad Turnbridge, Serra installe sa caméra sur un pont tournant ferroviaire qui cadre et balaye l'espace, renouant ainsi avec un dispositif qui évoque le cinéma des premiers temps. Comme cela se pratiquait couramment, la caméra, montée sur un appareil mobile, opère un mouvement panoramique sans se mouvoir elle-même (les déplacements effectifs de la caméra, quand Serra y recourt, vont
4 Sur ce point, voir les essais de Krauss centrés sur le montage dans les sculptures de Serra et sur leur dimension phénoménologique (Rosalind Krauss, "Redessiner la sculpture: Richard Serra", "Abaisser, étendre, contracter, comprimer, tourner: regarder l'œuvre de Richard Serra", L'originalité de l'avant-garde et autres mythes modernistes [1985], Paris, Macula, 1993, pp. 307-334 [traduit par JeanPierre Criqui]).

5 En 1969, Richard Serra projette Wawelength (Michael Snow, Etats-Unis, 1966-1967, 45') en Europe, dans le cadre de ses expositions personnelles (Annette Michelson, Richard Serra, Clara Weyergraf, "The Films of Richard Serra: An Interview ", in October, n' ${ }^{\circ}$ 10, automne 1979, repris dans Richard Serra, Ecrits et entretiens, Daniel Lelong Ed., Paris, 1990, p. 87 [traduit par Gille Courtois]).

6 Annette Michelson, Richard Serra, Clara Weyergraf, "The Films of Richard Serra: An Interview", op. cit., p. 100. 
$7 / d .$, p. 96.

$8 \mathrm{lbid}$.

9 /d., p. 124.

$10 / d .$, p. 94. d'ailleurs à l'encontre du mouvement du pont). La structure mobile en acier redouble le cadrage au sein du plan. De plus, elle autorise un jeu de variation sur la profondeur de champ: parfois, le paysage est cadré en plan moyen; à d'autres moments, il coïncide avec le point de fuite, seule l'armature latérale du pont saillant. Serra lui-même assimile Railroad Turnbridge à un film-dispositif, récusant la catégorie du documentaire sur l'architecture industrielle :

"Je ne suis pas allé filmer un pont parce que je pensais que c'était un objet industriel intéressant, ou une relique de l'Amérique authentique. Je crois qu'il m'était vraiment nécessaire d'approfondir ce que signifiait pour moi la 〈pontéité〉.» 7

La "pontéité», selon nous, a non seulement trait à la fonctionnalité d'une structure en acier modulable, mais encore à la découpe mécanique de l'espace qu'elle opère.

Selon Serra, le film s'impose comme une évidence lorsqu'il découvre le pont qui surplombe la rivière Willamette, dans la région de l'Oregon. Croisant le préposé au pont, il lui demande d'actionner le mécanisme. L'expérience, avec son caractère d'attraction, appelle la fixation filmique qui permet de percevoir autrement le mouvement du pont. Serra s'empresse d'aller chercher une caméra: sa première prise de vue devient le premier plan du film 8 ... Le tournage de Railroad Turnbridge s'étale sur une période d'un an, Serra se rendant à six reprises sur les lieux. Optant pour une position non interventionniste, il conçoit sur le même mode dépersonnalisé le montage du film, respectant la chronologie du mécanisme du pont tournant ("on le voit s'ouvrir, un bateau passe sur le côté, il se ferme, un train passe sur le pont», nous informe-t-il9).

Serra inscrit ostentatoirement Railroad Turnbridge, film suspendu à la fonctionnalité d'une machine, dans l'esthétique industrielle. Un gros plan sur les engrenages du pont, confinant à l'abstraction, emblématise cette dimension machiniste, qui évoque la structure grillagée du modulateur de lumière et d'espace de Laszlo Moholy-Nagy (Lichtspiel Schwarz-Weiss-Grau, Allemagne, 1930, 5' 30"). Et il est significatif que Serra invoque le modèle du Ballet mécanique (Fernand Léger, Dudley Murphy, France, 1924, 20') et la pratique cubiste du collage d'éléments industriels lorsque Michelson fait référence au pont tournant d'Octobre (S. M. Eisenstein, URSS, 1927), celle-ci concédant toutefois que le pathos du cheval qui tombe fait ici défaut ${ }^{\mathbf{1 0}}$. A notre sens, pourtant, le film de Serra ne peut pas être assimilé à une esthétique de la fragmentation, qui fait fond sur le gros plan et l'objet partiel, sur la variation des angles de vue et le montage court. 

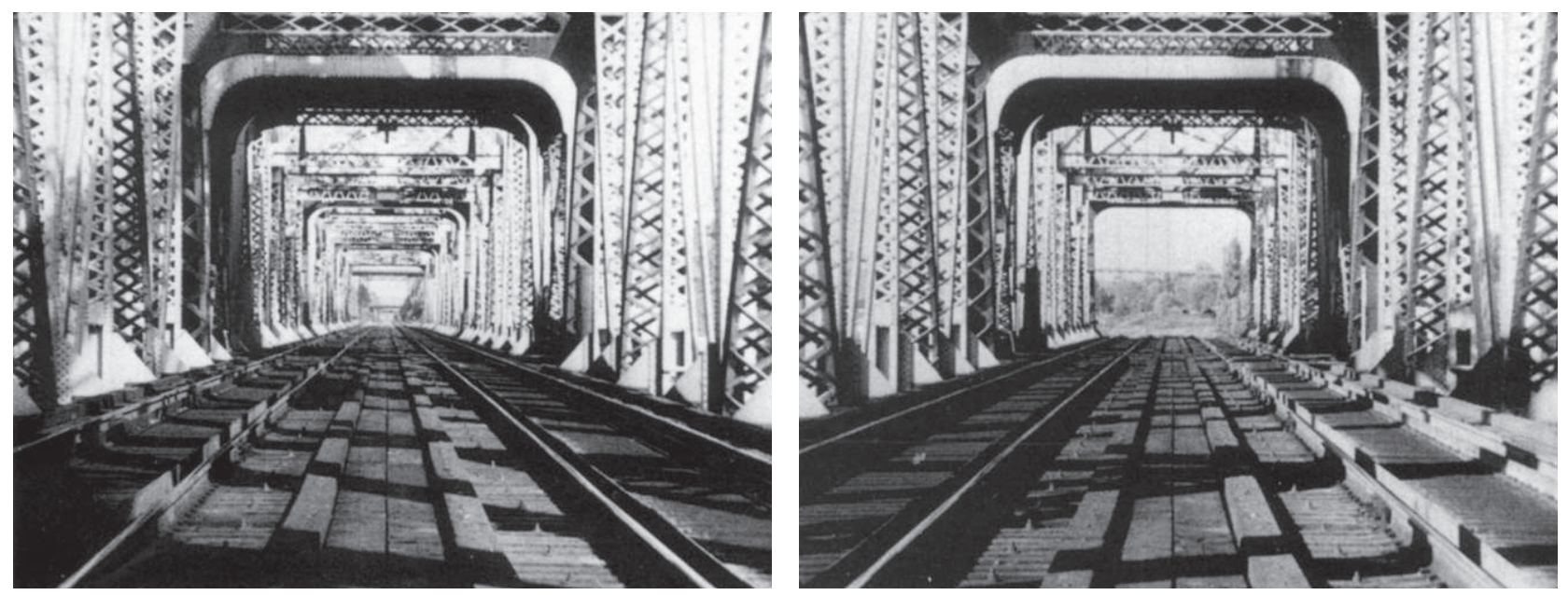

RAILROAD TURNBRIDGE (1975-1976)

Selon nous, l'exclusion d'une esthétique machiniste peut être ressaisie au plus près si nous rapportons Railroad Turnbridge à la vogue des documentaires architecturaux, dans les années 1920. René Clair avec $L a$ tour (France, 1928, 11') ou Joris Ivens avec Le pont (Pays-Bas, 1928, 11'), par exemple, participent d'un commun enthousiasme pour les nouveaux matériaux, en particulier le fer et l'acier. Si nous nous en tenons au motif du pont tournant, les pratiques d'Ivens et de Serra peuvent être opposées terme à terme. Serra opte pour l'esthétique du plan long, installant sa caméra au sein d'un dispositif qui détermine la forme du film. Au contraire, Ivens adopte un dispositif extrêmement léger: la caméra $16 \mathrm{~mm}$ portable la plus maniable de l'époque lui permet de multiplier les prises de vues, de manipuler et morceler le sujet filmé11. Serra, écartant le formalisme géométrique des symphonies visuelles et urbaines, souligne la spécificité et la singularité d'un site industriel, tout en s'inscrivant dans la postérité du minimalisme. Par ailleurs, nous pouvons relever la méfiance que Serra manifeste vis-à-vis d'Ivens, s'agissant d'un autre objet et contexte, c'est-à-dire des milieux de l'aciérie dans les années 1970. Dénonçant le film qu'Ivens a tourné dans une aciérie en Chine comme de la "propagande théâtrale», il renchérit:

"Cela semblait si artificiel, si maladroit. C'est un film dans la ligne du parti. Il n'y avait pas de description claire de l'aliénation dans ces usines.»12

Cette stigmatisation est au centre de Steelmill/Stablwerk (Richard Serra, Clara Weyergraf, Etats-Unis, 1979, 29'). Mais revenons aux dispositifs ferroviaires de Serra.

11 Joris Ivens expose en ces termes l'avantage de recourir à une caméra automatique, plus malléable qu'un appareil professionnel: "J'ai employé, à dessein, un petit appareil de prises de vue limité à vingt-cing mètres de film, pour être résolument libre de mes mouvements, et pouvoir tourner autour de l'objet et le saisir dans sa lumière extérieure indispensable dans l'enregistrement des documentaires " (cité dans Jean-Loup Passek (éd), Joris Ivens. Cinquante ans de cinéma, Centre Georges Pompidou, Paris, 1979, p. 18).

12 Annette Michelson, Richard Serra, Clara Weyergraf, "The Films of Richard Serra: An Interview ", op. cit., p. 130. Serra fait vraisemblablement allusion à L'usine des générateurs (131'), composant un volet du cycle Comment Yukong déplaça les montagnes (Joris Ivens, Chine, 1971-1975, 12h). 
RAILROAD TURNBRIDGE (1975-1976)

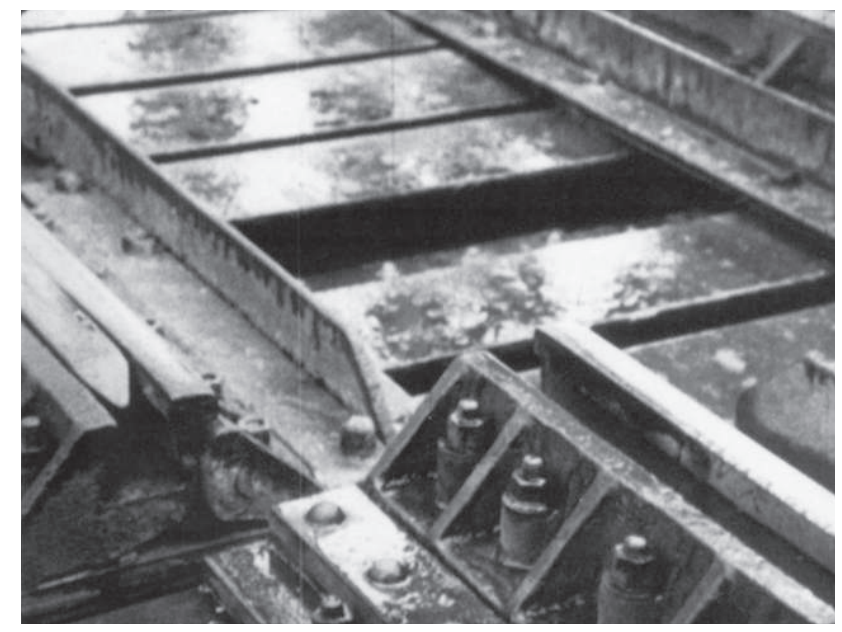

13 /d., p. 95.

\section{Une machine regarde une autre machine}

Dans le même entretien, Serra décrit précisément le mécanisme d'enchâssement qui façonne la structure de Railroad Turnbridge:

"[Le film] utilise le dispositif de la structure du tunnel pour cadrer le paysage, mais il se retourne aussi sur lui-même et se cadre luimême. L'illusion créée pose la question de ce qui se meut et de ce qui est fixe. Est-ce que c'est la caméra qui bouge et le pont qui est fixe, ou vice-versa?»13

Serra intente un procès de désubjectivation de la vision, amenant le spectateur à s'identifier au point de vue du pont tournant. Curieusement, cette impersonnalité de l'énonciation désoriente le spectateur, dépris de ses repères spatio-temporels. Au premier abord, la caméra semble adopter un point de vue ferroviaire (comme si elle était fixée à l'avant d'une locomotive, elle-même à l'arrêt): c'est le cas des débuts et des fins de plans sur la voie ferrée, lorsque les rails sont emboîtés. Pourtant, le point de vue d'un train ne concorde pas avec celui de la caméra - et lorsqu'un train vient effectivement à passer, la caméra le cadre en contre-plongée, évoquant certaines prises de vue récurrentes dans le cinéma soviétique des années 1920. Le lent mouvement de rotation du pont opacifie la représentation qui ne peut plus être rapportée à un point de vue à hau-

14 Serra déclare: "Je m'intéresse vraiment au noir et blanc. Quand j'ai tourné le film sur le pont tournant, presque tous les jours le soleil était obscurci par le brouillard. II n'y avait pas d'ombre. L'espace était donc aplati et compressé" (id., p. 127). teur d'homme. L'espace balayé subit un étrange effet d'aplatissement et d'étirement qui n'est pas étranger, comme le note Serra, à l'intensité de la lumière et à la qualité photographique du noir et blanc ${ }^{\mathbf{1 4}}$. L'effet d'indistinction entre le mouvement et la stase repose sur une superposition des points de vue: le spectateur confond le mouvement actuel de 
rotation du pont avec le déplacement illusoire de la caméra, voire avec le mouvement de l'espace environnant lui-même. Bref, comme le remarquait le Dr Saalschutz à propos d'un autre film mécanique, La marche des machines (Eugen Deslaw, France, 1929, 9'), le cinéma peut retranscrire à l'écran la confusion qui s'empare du voyageur, attribuant un mouvement au train immobile dans lequel il se tient alors que c'est le train qui lui fait face qui se met en branle $\mathbf{1 5}$.

A maints égards, le cinéma structurel fait ici figure de modèle, comme le reconnaît implicitement Serra en relevant l'influence qu'exercent sur lui les films expérimentaux de la fin des années 1960 et du début des années 1970. Mais il indique encore une autre piste de lecture qui nous paraît particulièrement féconde au regard des dispositifs ferroviaires : il rapporte le «contenu» de son film au "passage, entre 1905-1906 et 1925, dans la construction métallique, de la soudure au rivetage» qui a donné lieu à l'édification "d'extraordinaires structures en acier», construites "pour l'efficacité de leur support, et rien d'autre»16. La construction industrielle aux Etats-Unis, à suivre la remarque de Serra, se superpose au développement du cinéma : l'un et l'autre se rationalisent et se massifient, jusqu'à aboutir à la linéarisation des formes filmiques et à la fonctionnalité de l'architecture vernaculaire. A tout point de vue, Railroad Turnbridge est un film industriel - pourtant conçu et réalisé en dehors des structures dominantes de production et de diffusion. La relation spéculaire entre l'objet filmé et le sujet filmant (désauteurisé) n'échappe pas à Serra qui a pu déclarer:

"Dans deux films, Railroad Turnbridge et Steelmill/Stablwerk, la caméra se révèle comme une machine qui regarde une machine, que ce soit un pont ou une forge.»17

En effet, dans les deux cas, une machine (la caméra) regarde une autre machine. Mais les intentions divergent. Face au pont tournant, Serra fait l'expérience d'une vue pure qui se retourne sur elle-même et s'autoaffecte: en termes phénoménologiques, nous pourrions parler de perception de l'acte perceptif (en bref: d'autoperception). Le retournement de la vue est littéral: la caméra cadre d'abord l'espace depuis le bras mobile du pont; puis elle se retourne sur son centre de perception - elle cadre, depuis le bras immobile du pont cette fois, une structure en croix qui barre l'écran avant de se mettre en mouvement. Face à la forge, Serra remonte la chaîne de fabrication de l'acier qui sert à ses sculptures et dénonce l'exploitation des ouvriers. Documentaire militant, Steelmill/Stablwerk met à nu les conditions de travail dans l'industrie métallurgique en Allemagne, juste avant que n'éclate une grève générale qui paralysera le pays. La vue du pont n'excède pas le domaine de
$15 \mathrm{~L}$. Saalschutz, "Mechanisms of Cinema", in Close Up, novembre 1929, $\mathrm{n}^{\circ} 29$, pp. 359363 (réédition: Kraus Reprint, Liechtenstein, 1969).

16 Annette Michelson, Richard Serra, Clara Weyergraf, "The Films of Richard Serra: An Interview", op. cit., p. 59.

17 Entretien avec Bernard Lamarche-Vadel, mai 1980, dans Richard Serra, Ecrits et entretiens, op. cit., p. 149. 
18 Sur ce point, voir la communication de Tom Gunning au colloque portant sur le cinéma des premiers temps et l'avant-garde, organisé à Vienne par Sixpack, du 8 au 13 mars 2002 (www.sixpackfilm.com/archive/veranstaltung/ festivals/earlycinema/symposion/symposion gunning.html). l'attraction : il s'agit de faire l'expérience d'un regard mobile et irréalisant que nous pouvons rapporter aux attractions optiques et mobiles conçues pour l'Exposition universelle de Paris, en 190018.

\section{La vue ostentatoire de Ken Jacobs}

Dans deux films qui étaient originellement intégrés à la performance From Muybridge to Brooklyn Bridge, Ken Jacobs dérègle le regard du spectateur, inversant en quelque sorte le dispositif de soulignement du cadre mis en jeu par Serra. Exploitant le caractère d'attraction de la vue panoramique, il intervient sur de courtes bandes de voyage en chemin de fer. Dans The Georgetown Loop, il reprend un film de 1905, qu'il cite intégralement avant de le moduler, tandis que dans Disorient Express, il repart d'un film de 1906. Les films-source, conservés à la Library of Congress (archive où des copies de films tirées sur papier sont déposées pour des raisons de copyright, et ce jusqu'en 1912), sont "pluriponctuels»: le premier comporte une coupe franche, le second une coupe ainsi que plusieurs sautes plus localisées. Par la nature même du tournage (une caméra placée à l'avant d'une locomotive filme un train), l'image est discontinue et intermittente. Nous l'avons dit, les films auxquels a recours Jacobs sont de l'ordre de l'attraction, condensant les éléments modernes de la vitesse, de la mécanicité et du déplacement à travers la vue panoramique.

Mais le simple fait de faire défiler côte à côte deux copies conformes du film (dupliqué à la tireuse optique), disposées en une relation de miroir, affole le regard du spectateur. Poussant à son comble ce jeu de désorientation, Jacobs renverse l'image et inverse le sens de défilement des plans. Ces stratégies de trompe-l'œil, se portant à l'encontre de la limitation bidimensionnelle de l'écran, permettent tout à la fois de creuser la profondeur de champ et de sculpter le paysage: le mouvement du travelling avant, conjugué au jeu d'inversion et de renversement des

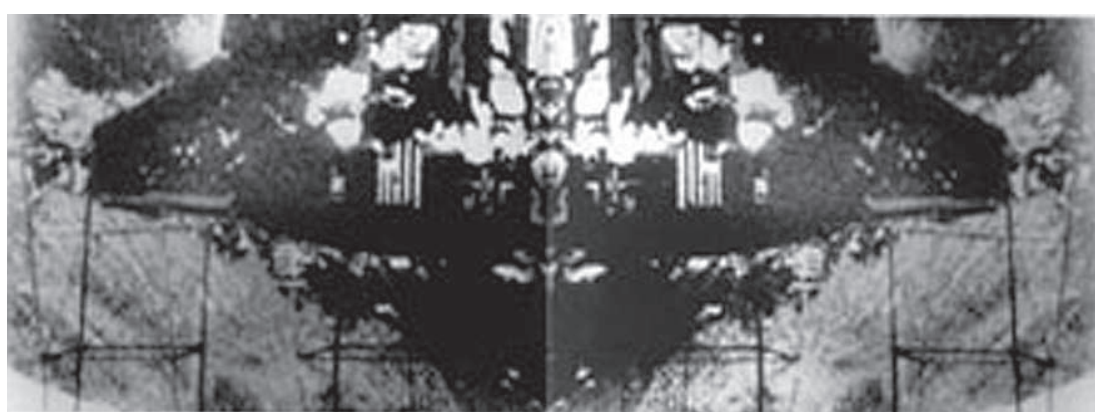


plans, impulse un effet tridimensionnel au paysage irréalisé, tout en faisant l'économie du recours effectif à la stéréoscopie. La vue-source, dont le caractère d'attraction s'est quelque peu estompé avec le temps, vire à l'abstraction: notre perception de la locomotion s'en trouve dénaturalisée (c'est le titre que Jacobs a donné à un autre volet de sa performance). Ajoutons que le cadre de la performance, autorisant une manipulation en direct, démultiplie les effets d'anamorphose de l'image, renouant avec la notion d'interprétation du film par le projectionniste (celui-ci déterminant par exemple la cadence du défilement des images).

Ready-made aidés, The Georgetown Loop et Disorient Express intentent un procès de subjectivation de la vision: le paysage qui défile s'apparente par moments à un miroitant test de Rohrschach; l'extrémité des deux plans communiquant au centre de l'écran, l'espace de la représentation devient un objet malléable qui défie les processus de la reconnaissance et de l'identification. Dans The Georgetown Loop, le train se plie et se déplie comme un accordéon, tandis que le paysage forme un dense cône énergétique, aux transformations incessantes, crevant l'écran. Les voyageurs agitent aux fenêtres des mouchoirs, signifiant, du moins après le passage de Jacobs, leur congé à une représentation filmique naturalisée (il est une autre lecture possible du film: les passagers saluent le déclin de l'ère industrielle, parcourant une dernière fois une ligne de chemin de fer, construite dans les années 1870 pour desservir les mines d'argent). Dans Disorient Express, le spectateur est d'emblée projeté dans un espace abstrait, le film-source n'étant donné dans son unicité qu'après coup, bloquant dès lors les mécanismes d'anticipation ou de remémoration de formes nommables. Le panorama de montagne s'en trouve transfiguré: il perd jusqu'à sa texture photographique, et devient prétexte à un éclatement kaléidoscopique des formes.

The Georgetown Loop et Disorient Express s'inscrivent dans la filiation des reprises de vues mobiles des premiers temps par des cinéastes et des artistes: nous pouvons convoquer comme antécédents le geste d'Ernie Gehr qui retravaille un panoramique primitif pris depuis un tramway (Eureka!) ou celui de Stan Douglas qui monte en boucle des plans Edison pris depuis l'avant d'un train et des extraits de La recherche $d u$ temps perdu de Proust récités sur la bande-son (Overture, Etats-Unis, 1986, boucle de quelques minutes). Mais les films de Jacobs se distinguent radicalement de ces phénomènes de reprises, à travers leurs effets kaléidoscopiques et stéréoscopiques. En un sens, ils sont plus proches de la démarche minimaliste du compositeur Steve Reich qui, dans Different Trains (1998), décline des bruits ferroviaires concrets (sifflements, passage du train sur une voie ferrée, etc.) 
jusqu'à former des scies et des motifs musicaux, organiquement mêlés à l'instrumentation.

Du dispositif de "surcadrage» mis en scène par Serra aux jeux d'anamorphose en 3-D de Jacobs, les films ferroviaires, dans le champ du cinéma expérimental, se définissent par la mobilité de leur regard et l'étrangeté de leur point de vue. Serra se concentre sur un motif qui s'efface en tant que pur support du train en temps normal: il découvre à travers le pont tournant une série de points de vue orientés et dirigés, répondant à une finalité non visuelle et non humaine. Serra donne ainsi forme à un découpage et à un quadrillage de l'espace qui évince tout opérateur humain. Jacobs appréhende la vue ferroviaire comme un objet trouvé qui recèle d'insoupçonnées qualités plastiques: projectile lancé à travers l'espace, le train anime le paysage, décompose sans visée analytique le mouvement et sculpte l'espace. L'échantillonnage de ces vues, citées in extenso (comme souvent dans le rapport particulier que Jacobs a instauré au found footage), permet de basculer sans crier gare dans le domaine du cinéma d'animation: les plis du paysage, la texture du grain de la pellicule et le défilement discontinu des photogrammes s'indifférencient.

\section{Annexes}

\section{Railroad Turnbridge (Richard Serra, Etats-Unis, 1975-1976, 16' 30")} Découpage:

plan 1) $0^{\prime} 8^{\prime \prime}$ / La caméra, fixe, est placée à l'arrière du bras mobile du pont tournant, suscitant un effet de creusement du champ redoublé par la vue en enfilade de la structure d'acier qui se confond au loin avec le point de fuite du plan; le pont se met en branle, balayant l'espace de droite à gauche après avoir déboîté les rails de la voie ferrée. Le cadrage du pont sur le paysage est très serré; avant que le pont tournant, au maximum de son ouverture, ne s'immobilise, nous apercevons un pont routier à l'arrière-plan.

plan 2) 3' 03" / La caméra, en un mouvement ascendant, cadre l'armature supérieure du pont, puis panote latéralement le long des cerceaux de l'armature (en une abrupte contre-plongée), avant de redescendre jusqu'au mécanisme du pont tournant, lui-même en rotation. La caméra, avant de s'immobiliser, se déplace vers la gauche, contredisant le mouvement du pont tournant vers la droite. Le cadrage du pont sur l'extérieur est large (la profondeur de champ sur la structure en acier de la balustrade est très réduite). Le pont tournant se referme et s'immobilise.

plan 3) 5' 30" / Le pont tournant est filmé de nuit, fermé, des lumières miroitant au loin.

plan 4) 6' 30" / La caméra cadre la voie ferrée, creusant le champ. Le pont tournant, à l'avant-plan, ainsi que quatre ponts routiers, à l'arrièreplan, enjambent la voie ferrée. II n'y a pas de mouvements, si ce n'est le déplacement des voitures, interne au plan. plan 5) 7' 45" / Un train traverse le pont tournant, passe au-dessus de la caméra, crevant le champ de l'arrière à l'avant-plan.

plan 6) 8' 15" / La caméra cadre en très gros plan et en contre-plongée le mécanisme de glissière qui sort du rail situé à l'avant-plan et se rétracte dans celui à l'arrière-plan. Le pont tournant se met en branle (8' 50"), amorçant un mouvement vers la droite (le plan est coupé avant que le paysage n'apparaisse à travers un cadrage large).

plan 7) 9' $00^{\prime \prime}$ / La caméra cadre en très gros plan les rouages dentés du mécanisme en mouvement du pont (le plan est à la limite de l'abstraction).

plan 8) 9' 48" / La caméra, sur le bras en mouvement du pont tournant, cadre en plan large le paysage (la profondeur de champ sur la structure en acier de la balustrade étant réduite). Au terme de son mouvement de balayement de gauche à droite, le pont tournant se referme.

plan 9) 10' 12" / Placée en position de contre-champ, sur le bras immobile du pont, la caméra cadre la partie supérieure d'un paquebot qui traverse le pont tournant ouvert (une structure en croix, à l'extrémité du bras mobile, barre l'écran). En face de la caméra, le bras du pont tournant se referme, en un mouvement de rotation vers la droite (11'25'). Le plan est coupé avant que le pont tournant ne se soit entièrement refermé.

plan 10) 12 ' $54^{\prime \prime}$ / Le bras mobile du pont tournant est filmé en plongée. Le pont tournant s'ouvre, en un mouvement de rotation de droite à gauche.

plan 11) $13^{\prime} 02^{\prime \prime}$ / La caméra filme en très gros plan l'extrémité 
inférieure des roues d'un train lancé à vive allure, balayant l'écran de droite à gauche.

plan 12) $13^{\prime} 20^{\prime \prime}$ / La caméra accompagne à travers un panoramique l'extrémité du bras en mouvement du pont tournant qui se referme, de gauche à droite. Le paysage, malgré le cadrage large, est peu distinct. plan 13) 13' 55"' - 16' / La caméra, à l'arrière du bras immobile du pont tournant, cadre le mouvement de rotation du bras en face, ouvert à son amplitude maximale (sa structure en croix barrant l'écran). Le pont tournant se referme, en un mouvement de rotation de gauche à droite.

\section{The Georgetown Loop (Ken Jacobs, Etats-Unis, 1996, 10')}

Source: The Library of Congress Paper Print Collection; film anonyme, 1905

Description de la vue d'origine: Deux plans, 1' 56". La caméra est placée à l'avant d'une locomotive lancée à vive allure. Elle filme un train, comportant quatre wagons, qui apparaît et disparaît alternativement à l'écran, au gré des contours. Les passagers, sur la plate-forme arrière du train et aux fenêtres, agitent des mouchoirs. Le plan embraye sur un pont suspendu où circule un train. Après $35^{\prime \prime}$, le train traverse un village, probablement Georgetown. Une coupe intervient lorsque le train quitte le village (après $\left.1^{\prime} 10^{\prime \prime}\right)$. Juste avant que le plan ne prenne fin, la distance se creuse entre la caméra et le train: la locomotive sort presque du cadre et le wagon de queue apparaît nettement comme disjoint de l'espace qu'occupe la caméra.

Modes de permutations: 1/ La vue défile sur la moitié droite de l'écran. 2/ La vue, dédoublée, défile synchroniquement, mais elle est dupliquée avec un effet de miroir. 3/ Idem. 4/ La vue, dupliquée, est renversée et défile synchroniquement à l'envers (le train, apparaissant cul pardessus tête, recule). 5/ Idem.
Disorient Express (Ken Jacobs, Etats-Unis, 1996, 29')

Source: The Library of Congress Paper Print Collection; A Trip Down Mount Tamalpais, film anonyme, 1906

Description de la vue d'origine: Deux plans (et quelques coupes), 5' 40". La caméra est à l'avant d'une locomotive. Elle filme un train, avec deux wagons, qui circule dans les montagnes. Le train (surtout la locomotive et le premier wagon) est partiellement masqué par les tournants. Une personne se tient sur les bas-côtés de la voie ( $\left.0^{\prime} 41^{\prime \prime}\right)$. L'image vire au blanc (2' $\left.04^{\prime \prime}-2^{\prime} 19^{\prime \prime}\right)$. Le train traverse un tunnel (3'56"'- 4' 09'). Une coupe intervient, alors que le train est masqué par un contour ( $\left.3^{\prime} 28^{\prime \prime}\right)$. Deux légère coupes suivent, provoquant un effet de saute de l'image (3' 57'" : le train entre dans le tunnel; 4' 04"': le train où se situe la caméra traverse à son tour le tunnel). Au sortir du tunnel, une personne salue le train, à droite de la voie. Puis apparaissent d'autres gens. L'image vire au noir, comme si le train se trouvait dans un tunnel (4'39'- $\left.4^{\prime} 44^{\prime \prime}\right)$. Le train croise un baraquement

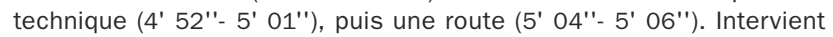
une nouvelle saute de l'image ( $\left.5^{\prime} 13^{\prime \prime}\right)$. Le train traverse une route et entre dans un village (5'17'"- 5' 19'). Un homme marche le long des voies, un autre les traverse précipitamment (5' 19'"- 5' 29"). Le train entre en gare, une foule s'ammassant sur le quai pour y prendre place (5' 32'" - 5' 40"').

Modes de permutations: $1 /$ La vue, dédoublée avec un effet de miroir, défile à l'envers et ses images sont inversées. 2/ La vue défile sur la moitié gauche de l'écran. 3/ La vue, dont les images sont inversées, défile à l'envers sur la moitié droite de l'écran. 4/ La vue, dédoublée avec un effet de miroir, défile à l'envers et ses images sont inversées. 5/ La vue, dédoublée avec un effet de miroir, défile à l'endroit et ses images sont inversées. 